\title{
A Comparative Study of Lattice Dynamics of Three- and Two-Dimensional $\mathrm{MoS}_{2}$
}

\author{
C. Ataca, ${ }^{+, \neq}$M. Topsakal, ${ }^{\ddagger}$ E. Aktürk, ${ }^{\neq}$and S. Ciraci* ${ }^{*+, \neq}$ \\ ${ }^{\dagger}$ Department of Physics and ${ }^{\ddagger}$ UNAM-Institute of Materials Science and Nanotechnology, Bilkent University, Ankara 06800, Turkey
}

ABSTRACT: This paper presents a comparative study of the lattice dynamics of three-dimensional layered $\mathrm{MoS}_{2}$ and twodimensional single layer $\mathrm{MoS}_{2}$ based on the density functional theory. A comprehensive analysis of energetics and optimized structure parameters is performed using different methods. It is found that the van der Waals attraction between layers of three-dimensional (3D) layered $\mathrm{MoS}_{2}$ is weak but is essential to hold the layers together with the equilibrium interlayer spacing. Cohesive energy, phonon dispersion curves, and corresponding density of states and related properties, such
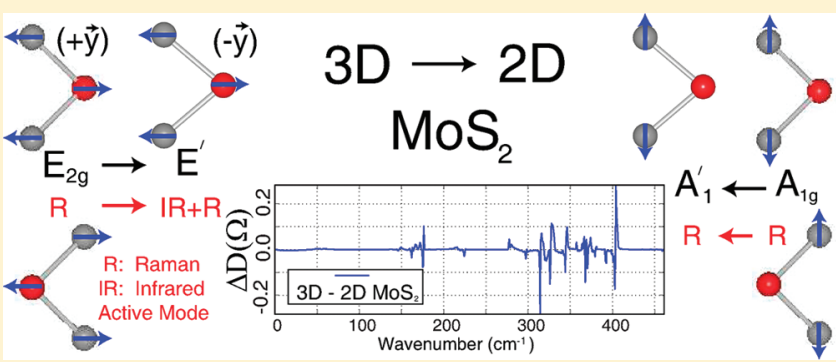
as Born-effective charges, dielectric constants, Raman and infrared active modes are calculated for 3D layered as well as 2D single layer $\mathrm{MoS}_{2}$ using their optimized structures. These calculated values are compared with the experimental data to reveal interesting dimensionality effects. The absence of a weak interlayer interaction in $2 \mathrm{D}$ single layer $\mathrm{MoS}_{2}$ results in the softening of some of Raman active modes.

\section{INTRODUCTION}

Three-dimensional (3D) $\mathrm{MoS}_{2}$, a well-known transitionmetal dichalcogenide, has two different stable structures: 3R$\mathrm{MoS}_{2}$ polytype ${ }^{1}$ and layered $2 \mathrm{H}-\mathrm{MoS}_{2}$. The latter consists of the stacking of $\mathrm{MoS}_{2}$ layers and is the subject matter of the present study. Various properties of $2 \mathrm{H}-\mathrm{MoS}_{2}$ (refs 2-17), in particular lattice dynamics and electronic energy band structure, have been studied extensively. Recently $2 \mathrm{D}$ suspended single layer $\mathrm{MoS}_{2}$ sheets, i.e., $1 \mathrm{H}-\mathrm{MoS}_{2}$ having hexagonal lattice have been produced. ${ }^{18-21}$ Single layer $\mathrm{MoS}_{2}$ nanocrystals of $\sim 30 \AA$ width were also synthesized on the $\mathrm{Au}(111)$ surface $^{22}$ and the first direct real space STM images of single layer $\mathrm{MoS}_{2}$ nanosheets have been reported. In the meantime, theoretical studies (refs 16 and $23-30$ ) on $1 \mathrm{H}-\mathrm{MoS}_{2}$ have appeared. Three-dimensional $2 \mathrm{H}-\mathrm{MoS}_{2}$ and $2 \mathrm{D} 1 \mathrm{H}-\mathrm{MoS}_{2}$, ${ }^{30}$ quasi-1D nanotubes, ${ }^{25}$ and nanoribbons ${ }^{16,26,29}$ of $\mathrm{MoS}_{2}$ share the honeycomb structure and display interesting dimensionality effects.

Properties of $\mathrm{MoS}_{2}$ nanocrystals are explored in diverse fields, such as nanotribology, ${ }^{31}$ hydrogen production, ${ }^{32,33}$ hydrodesulfurization catalyst used for removing sulfur compounds from oil, ${ }^{34-40}$ solar cells, ${ }^{41}$ and photocatalysis. ${ }^{42}$ Triangular $\mathrm{MoS}_{2}$ nanocrystals of diverse sizes were investigated using atom-resolved scanning tunneling microscopy. ${ }^{43}$ A superlow coefficient of sliding friction between surfaces coated with $1 \mathrm{H}-\mathrm{MoS}_{2}$ has been measured recently. ${ }^{44} \mathrm{~A}$ transistor fabricated from the single layer $\mathrm{MoS}_{2}$ has heralded the features of $1 \mathrm{H}-\mathrm{MoS}_{2}$, which is superior to graphene. ${ }^{45}$ Studies to date suggest that $\mathrm{MoS}_{2}$ sheets can be promising for optoelectronic devices, solar cells, and LEDs. Most recently, the Raman spectra of $\mathrm{MoS}_{2}$ sheets have been measured as a function of their thickness. $^{21,46}$
Despite the fact that $2 \mathrm{H}-\mathrm{MoS}_{2}$ is a layered material, where $\mathrm{MoS}_{2}$ layers were bound by weak interlayer interaction, significant dimensionality effects have been observed. For example, while $3 \mathrm{D} \mathrm{MoS}_{2}$ is an indirect band gap semiconductor, the band gap increases and becomes direct in $2 \mathrm{D}$ single layer $\mathrm{MoS}_{2}{ }^{20}$ This dimensionality effect may lead to photoluminescence applications in nanoelectronics. ${ }^{47}$ While the lattice dynamics of $2 \mathrm{H}-\mathrm{MoS}_{2}$ have been studied actively in the past by using inelastic neutron scattering and Raman-infrared spectroscopy ${ }^{6,24}$ and its phonon dispersion curves, phonon density of states, infrared and Raman active modes are calculated in terms of force constants derived from experimental data, yet an ab initio treatment including van der Waals interaction (vdW) is absent. Recent papers ${ }^{21,46}$ investigating the Raman spectra of $3 \mathrm{D}$ and $2 \mathrm{D} \mathrm{MoS}_{2}$ came up with conflicting conclusions. In this paper, we present our theoretical investigation of the lattice dynamics and related properties of $2 \mathrm{H}$ - and $1 \mathrm{H}-\mathrm{MoS}_{2}$. Our study is carried out from the first principles within van der Waals (vdW) density functional theory (DFT), where atomic structure, lattice constants, and relevant energetics are obtained from structure optimization including vdW correction. This method provides a proper treatment of the interaction between the layers of $2 \mathrm{H}-\mathrm{MoS}_{2}$, as well as their spacings. Finally, calculated properties of $2 \mathrm{H}-$ and $1 \mathrm{H}-\mathrm{MoS}_{2}$, such as Raman and infrared active modes, bulk modulus, dielectric constants, and effective charges are compared to reveal dimensionality effects. Even though the calculated

Received: $\quad$ May 31, 2011

Revised: July 18, 2011

Published: July 22, 2011 


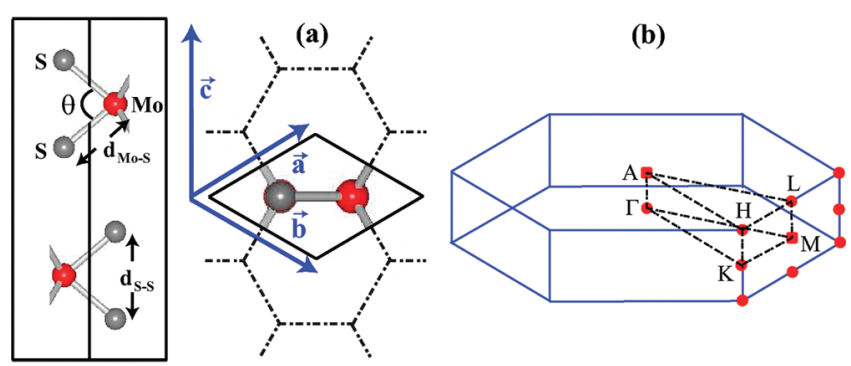

Figure 1. (a) Side and top views of atomic structure of $2 \mathrm{H}-\mathrm{MoS}_{2}$ with hexagonal lattice. The unit cell is delineated, lattice constants $|a|=|b|, c$ and internal structure parameters are indicated. Honeycomb structure consisting of Mo (red ball) and $\mathrm{S}_{2}$ (gray balls) located at the corners of hexagons is seen in the top view. (b) Corresponding Brillouin zone with symmetry directions.

interlayer interaction is a weak vdW, its effects on lattice dynamics are significant. We showed that the calculated shifts of the frequencies of Raman active modes as a result of phonon softening upon lowering the dimensionality from $3 \mathrm{D}$ to a $2 \mathrm{D}$ single layer $\mathrm{MoS}_{2}$ are sensitive to the deviation of lattice constants from the experimental values.

\section{METHOD}

We carried out first-principles plane wave calculations within DFT and used ultrasoft pseudopotential (UP). ${ }^{48}$ The exchange correlation potential is approximated by a generalized gradient approximation (GGA) using the PW91 ${ }^{49}$ functional for both spin-polarized and spin-unpolarized cases. The vdW corrections ${ }^{50,51}$ are included to determine the interlayer spacing in $2 \mathrm{H}-\mathrm{MoS}_{2}$. While all discussions in the paper are based on the results obtained within GGA combined with vdW interaction, the calculations within local density approximation $^{52}$ (LDA) using UP are also performed for the purpose of comparison. All structures are treated using the periodic boundary conditions. Kinetic energy cutoff, Brillouin zone (BZ) sampling is determined after extensive convergence analysis. A large spacing of $\sim 10 \AA$ between $2 \mathrm{D}$ single layers of $\mathrm{MoS}_{2}$ is taken to prevent interactions between them. A plane-wave basis set with kinetic energy cutoff of $952 \mathrm{eV}$ is used for high accuracy of the results. ${ }^{53}$ In the self-consistent field potential and total energy calculations $\mathrm{BZ}$ is sampled by special k-points. ${ }^{54}$ The numbers of these k-points are $(15 \times 15 \times 7)$ for $2 \mathrm{H}-\mathrm{MoS}_{2}$ and $(25 \times 25 \times 1)$ for $1 \mathrm{H}-\mathrm{MoS}_{2}$, respectively. All atomic positions and lattice constants are optimized by using the conjugate gradient method, where the total energy and atomic forces are minimized. The convergence for energy is chosen as $10^{-7} \mathrm{eV}$ between two consecutive steps, and the maximum Hellmann-Feynman force acting on each atom is less than $10^{-5} \mathrm{eV} / \AA ̊$ upon ionic relaxation. The pressure in the unit cell is kept below $1 \mathrm{kbar}$. Numerical calculations have been performed by using the PWSCF package. ${ }^{55}$ The phonon dispersion curves are calculated within density functional perturbation theory (DFPT) using plane wave methods as implemented in the PWSCF package.

\section{ENERGETICS AND OPTIMIZED STRUCTURES OF 3D} AND 2D MOS 2

Three dimensional bulk $2 \mathrm{H}-\mathrm{MoS}_{2}$ is a stable layered structure and includes two $\mathrm{MoS}_{2}$ units in the unit cell. Each layer consists of a monatomic Mo plane between two monatomic $S$ planes having the same $2 \mathrm{D}$ hexagonal lattice. Mo and $\mathrm{S}_{2}$ occupy alternating corners of a hexagon to form a honeycomb structure. Each Mo has six nearest $S$ atoms, and each $S$ atom has three nearest Mo atoms. Two layers in the unit cell are displaced relative to each other, as such that Mo atoms of one layer are situated on top of $S$ atoms in two adjacent layers. In this respect, the arrangements of layers are different from that of graphite, where three carbon atoms of one layer are located above the hollow sites (center of the hexagon) of the adjacent layers. The atomic configurations and relevant structural parameters of $3 \mathrm{D}$ $2 \mathrm{H}-\mathrm{MoS}_{2}$ are illustrated in Figure 1a. First-principles calculations of lattice dynamics and related properties of $3 \mathrm{D}$ and $2 \mathrm{D}$ $\mathrm{MoS}_{2}$ are obtained by using the optimized atomic structure. Therefore, we start to investigate the energetics, minimum energy atomic structure, and structure parameters of these crystals.

\section{口 OPTIMIZED STRUCTURE}

The interaction between $1 \mathrm{H}-\mathrm{MoS}_{2}$ layers in $2 \mathrm{H}-\mathrm{MoS}_{2}$ has predominantly vdW character. Therefore, DFT calculations within GGA but without vdW are known to overestimate the interlayer distance and the lattice parameter $c$ in Figure 1a. To present a correct estimation of lattice constants, we included vdW correction to GGA calculations using two different methods. The first one $(\mathrm{GGA}+\mathrm{D})$ is used mainly for molecules and corrects GGA by adding interatomic $C_{6} / R^{6}$ interaction. The $C_{6}$ coefficient and cutoff distance are deduced from relevant molecules. ${ }^{50}$ The second method (GGA+DF) aims at solutions from the first principles without empiricism and uses nonlocal exchange-correlation functional to treat $\mathrm{vdW}$ interaction in GGA. ${ }^{51}$ The latter method is tested for molecules and solids. In order to find the most appropriate method, we carried out GGA calculations without vdW correction, as well as GGA+D and GGA+DF calculations. For the sake of comparison, we also performed LDA calculations, which is known to include vdW interaction partially. ${ }^{56,57}$ In our analysis we consider two layered 3D crystals both having honeycomb structure, namely, graphite and $2 \mathrm{H}-\mathrm{MoS}_{2}$, in which the cohesions of layers are known to be achieved mainly by the weak vdW interaction. The optimized lattice constants $|a|=|b|$ and $c$, and interlayer interaction energy $E_{\mathrm{i}}$ calculated by using these methods are presented in Table 1. For comparison the experimental values are also given. The interlayer interaction energy or cohesion of $2 \mathrm{H}-\mathrm{MoS}_{2}$ relative to individual $\mathrm{MoS}_{2}$ layers can be deduced by calculating the total energy of $2 \mathrm{H}-\mathrm{MoS}_{2}$ as a function of interlayer spacing $z$ in the direction perpendicular to the $\mathrm{MoS}_{2}$ layer, i.e., $E_{\mathrm{T}}(z)$ and by setting $E_{\mathrm{T}}(z \rightarrow \infty)=0$. Then the absolute value of half of the minimum of $E_{\mathrm{T}}(z=c)$ is taken as $E_{\mathrm{i}}$ (interlayer interaction energy per layer).

For graphite, GGA without vdW attains the experimental $a$, but overestimates $c$ by $25 \%$ relative to the experimental value. ${ }^{58-62}$ Expectantly, the calculated interlayer interaction energy $E_{i}=5 \mathrm{meV}$ per layer is much smaller than the experimental value. ${ }^{58-62}$ On the other hand, the values of $c$ and $E_{i}$ calculated for graphite are improved significantly when $\mathrm{vdW}$ correction is included. While GGA+DF overestimates $c$ by $6.6 \%$, GGA+D underestimates it by $4.2 \%$. However, both methods result overestimate $E_{i}$ by $14 \%$ relative to experimental value. Interestingly, LDA yield almost the experimental value of $c$, even if it underestimates the experimental $E_{i}$ by $8 \%$. 
Table 1. Lattice Constants $|a|=|b|$ and $c$, and Interlayer Interaction Energy $E_{\mathrm{i}}$ (per graphene $\left(\mathrm{C}_{2}\right)$ or $\mathrm{MoS}_{2}$ unit) of Graphite and $2 \mathrm{H}-\mathrm{MoS}_{2}$ Calculated Using GGA, GGA+D and GGA+DF, and LDA Methods. ${ }^{a}$

\begin{tabular}{|c|c|c|c|c|c|c|}
\hline & \multicolumn{3}{|c|}{ graphite (graphene) } & \multicolumn{3}{|c|}{$2 \mathrm{H}-\mathrm{MoS}_{2}\left(1 \mathrm{H}-\mathrm{MoS}_{2}\right)$} \\
\hline & $a(\AA)$ & $c(\AA)$ & $E_{\mathrm{i}}\left(\mathrm{eV} / \mathrm{C}_{2}, \mathrm{kcal} / \mathrm{mol}\right)$ & $a(\AA)$ & $c(\AA)$ & $E_{\mathrm{i}}\left(\mathrm{meV} / \mathrm{MoS}_{2}, \mathrm{kcal} / \mathrm{mol}\right)$ \\
\hline GGA & $2.461(2.463)$ & 8.407 & $5,0.115$ & $3.215(3.214)$ & 15.540 & $6,0.138$ \\
\hline $\mathrm{GGA}+\mathrm{D}$ & $2.461(2.463)$ & 6.425 & $122,2.816$ & $3.220(3.220)$ & 12.411 & $160,3.693$ \\
\hline $\mathrm{GGA}+\mathrm{DF}$ & $2.463(2.463)$ & 7.157 & $116,2.678$ & $3.258(3.254)$ & 13.152 & $176,4.063$ \\
\hline $\mathrm{LDA}+\mathrm{UP}$ & $2.441(2.441)$ & 6.669 & $96,2.216$ & $3.125(3.118)$ & 12.137 & $110,2.539$ \\
\hline experiment & $2.461-2.463^{58-62}(2.455)$ & $6.708-6.712^{58-62}$ & $104 \pm 10,^{58,62}$ & $3.16^{10,63,64}$ & $12.29,,^{10,63}$ & $140 \pm 22,^{67}$ \\
\hline & & & $2.401 \pm 0.231$ & $\left(3.20{ }^{65} 3.27^{66}\right)$ & $12.30^{64}$ & $3.239 \pm 0.498$ \\
\hline
\end{tabular}

${ }^{a}$ The corresponding values calculated for graphene and single layer $\mathrm{MoS}_{2}$ are given in parentheses. Experimental values are given for the sake of comparison. Experimental values of lattice constant $a$ of $1 \mathrm{H}-\mathrm{MoS}_{2}$ given by refs 65 and 66 appear to be too large and not confirmed.

Table 2. Lattice Constants $|a|=|b|$ and $c$, Internal Structure Parameters, Such as Bond Lengths $d_{\mathrm{Mo}-\mathrm{S}}$ and $d_{\mathrm{S}-\mathrm{S}}$, S-Mo-S Bond Angles $\theta(\mathrm{S}-\mathrm{Mo}-\mathrm{S})$, Bulk Modulus $B_{0}$, in-Plane Stiffness $C$ for $2 \mathrm{D}$ Single Layer, Cohesive Energy per $\mathrm{MoS}_{2}$ Unit $E_{\mathrm{C}}$, Born Effective Charges of Constituent Atoms $Z_{\mathrm{B}}{ }^{*}[\mathrm{Mo}]$ and $Z_{\mathrm{B}}{ }^{*}[\mathrm{~S}]$, High Frequency Intralayer and Interlayer Dielectric Constants, $\varepsilon$ and $\varepsilon_{\perp}$ Calculated for $2 \mathrm{H}-\mathrm{MoS}_{2}$ and $1 \mathrm{H}-\mathrm{MoS}_{2}$ Using the GGA+D Method

\begin{tabular}{lccccccccccc} 
& $a(\AA)$ & $c(\AA)$ & $d_{\mathrm{Mo}-\mathrm{S}}(\AA)$ & $d_{\mathrm{S}-\mathrm{S}}(\AA)$ & $\Theta$ & $\mathrm{B}_{0}(\mathrm{GPa}) / \mathrm{C}(\mathrm{N} / \mathrm{m})$ & $E_{\mathrm{C}}(\mathrm{eV})$ & $Z_{\mathrm{B}}{ }^{*}(\mathrm{Mo})$ & $Z_{\mathrm{B}}{ }^{*}(\mathrm{~S})$ & $\varepsilon_{\|}$ & $\varepsilon_{\perp}$ \\
$2 \mathrm{H}-\mathrm{MoS}_{2}$ & 3.220 & 12.411 & 2.436 & 3.150 & 80.564 & 44 & 15.316 & 1.23 & -0.57 & 15.60 & 6.34 \\
$1 \mathrm{H}-\mathrm{MoS}_{2}$ & 3.220 & & 2.437 & 3.153 & 80.617 & 145.82 & 15.156 & 1.21 & -0.57 & 4.58 & 1.26 \\
\hline
\end{tabular}

An analysis made for $2 \mathrm{H}-\mathrm{MoS}_{2}$ reveals similar trends and indicates vdW interaction as the dominant interaction between its layers. While interlayer interaction calculated with GGA is only $6 \mathrm{meV}$ per $\mathrm{MoS}_{2}$ unit, $c$ is badly overestimated to be $15.54 \AA$. This is $26.4 \%$ longer relative to the experimental value of the lattice constant $c$ measured $^{10,63,64}$ to be $c=12.29-12.30 \AA$. While GGA optimizes lateral lattice constants at $|a|=|b|=$ $3.215 \AA$, the measured lateral lattice constants $|a|=|b|=$ $3.16 \AA^{10,63,64}$ In contrast, GGA+D and GGA+DF methods estimate $E_{\mathrm{i}}$ to be 160 and $176 \mathrm{meV}$, respectively. Accordingly, $c$ values calculated by these vdW corrections are $12.41 \AA$ (i.e., overestimated by $0.9 \%$ ) and $13.15 \AA$ (overestimated by $7.0 \%$ ), respectively. The lateral lattice constant $a$ is optimized by GGA $+\mathrm{D}$ to be $\sim 3.22 \AA$ (3.258 $\AA$ by GGA+DF). On the other hand, LDA underestimates both $a$ and $c$ by $\sim 2 \%$ relative to experimental value and predicts $E_{\mathrm{i}}=110 \mathrm{meV}$. In view of this analysis and comparison with measured ${ }^{10,63,64}$ lattice constants, the GGA+D method appears to be suitable to obtain optimized structure and related properties of $2 \mathrm{H}-\mathrm{MoS}_{2}$ and $1 \mathrm{H}-\mathrm{MoS}_{2}$. For the rest of the paper, we will use results obtained from this method unless it is stated otherwise.

\section{COHESIVE ENERGY AND OTHER PROPERTIES}

The cohesive energy per $\mathrm{MoS}_{2}$ unit relative to the free Mo and $S$ atoms, $E_{\mathrm{C}}=-E_{\mathrm{T}}[$ Crystal $]+E_{\mathrm{T}}[\mathrm{Mo}]+2 E_{\mathrm{T}}[\mathrm{S}]$, is calculated from the structure optimized total energies of the $3 \mathrm{D}$ crystal, $E_{\mathrm{T}}\left[2 \mathrm{H}-\mathrm{MoS}_{2}\right] / 2$ or $2 \mathrm{D}$ crystal $E_{\mathrm{T}}\left[1 \mathrm{H}-\mathrm{MoS}_{2}\right]$ and the free atom total energies of Mo and $S, E_{\mathrm{T}}[\mathrm{Mo}]$ and $E_{\mathrm{T}}[\mathrm{S}]$, respectively. The calculated values of $E_{\mathrm{C}}$ for $2 \mathrm{H}-\mathrm{MoS}_{2}$ and $1 \mathrm{H}$ $\mathrm{MoS}_{2}$ are 15.316 and $15.156 \mathrm{eV}$ per $\mathrm{MoS}_{2}$ unit, respectively. Their difference is exactly the interlayer interaction energy of 3D $\mathrm{MoS}_{2}$, which was calculated to be $160 \mathrm{meV}$. This indicates that the cohesion of the layers in $2 \mathrm{H}-\mathrm{MoS}_{2}$ is the same as the cohesion of $1 \mathrm{H}-\mathrm{MoS}_{2}$. In addition to cohesive energy, the zero pressure bulk modulus $B_{0}$, is an important mechanical property of $3 \mathrm{D}$ crystals. Here we calculated the bulk modulus of
$2 \mathrm{H}-\mathrm{MoS}_{2}$ by fitting the Murnaghan equation ${ }^{68}$ as $44 \mathrm{GPa}$. The experimental value ${ }^{69}$ is given as $43 \mathrm{GPa}$. Using van der Waals included DFT Rydberg et al. ${ }^{11}$ calculated $B_{0}$ as $39 \mathrm{GPa}$. Our value calculated for the bulk modulus of $B_{0}$ is in good agreement with the experimental value.

Single layer, $1 \mathrm{H}-\mathrm{MoS}_{2}$ has high planar strength but transversal flexibility. While Young's modulus normally characterizes the mechanical strength of bulk materials, owing to the ambiguities in defining the Young's modulus for the $2 \mathrm{D}$ honeycomb structure, one can use in-plane stiffness $C=\left(1 / A_{0}\right)\left(\partial^{2} E_{S} / \partial \varepsilon_{\mathrm{s}}^{2}\right)$ in terms of the equilibrium area of the $2 \mathrm{D}$ cell, $A_{0}{ }^{70,71}$ We focused our attention on the harmonic range of the elastic deformation, where the structure responded to strain $\varepsilon$ linearly. Here $\varepsilon_{\mathrm{s}}$ is the elongation per unit length. The strain energy is defined as $E_{\mathrm{S}}=$ $E_{\mathrm{T}}\left(\varepsilon_{\mathrm{s}}\right)-E_{\mathrm{T}}\left(\varepsilon_{\mathrm{s}}=0\right)$; namely, the total energy at a given strain $\varepsilon_{\mathrm{s}}$ minus the total energy at zero strain. The calculated in-plane stiffness of $1 \mathrm{H}-\mathrm{MoS}_{2}$ is $145.82 \mathrm{~N} / \mathrm{m}$. This value can be compared with the experimental value of graphene, i.e., $340 \pm 50 \mathrm{~N} / \mathrm{m}^{72}$

Sun et al. ${ }^{17}$ obtained high-frequency dielectric constants, $\varepsilon$, and Born effective charge, $Z_{\mathrm{B}}{ }^{*}$, of $2 \mathrm{H}-\mathrm{MoS}_{2}$ by fitting to the experimental data. They found Born effective charges, $Z_{\mathrm{B}}{ }^{*}[\mathrm{Mo}]=1.11$ electrons (positive charge) for Mo and $Z_{\mathrm{B}}{ }^{*}[\mathrm{~S}]=-0.52$ electrons (negative charge) for each $S$ atom and dielectric constants $\varepsilon_{\|}=$ 15.2 and $\varepsilon_{\perp}=6.2$ in the intralayer and interlayer directions, respectively. Here we calculate Born effective charges and highfrequency dielectric constant of $2 \mathrm{H}-\mathrm{MoS}_{2}$ to be $Z_{\mathrm{B}}{ }^{*}[\mathrm{Mo}]=1.23$ electrons and $Z_{\mathrm{B}}{ }^{*}[\mathrm{~S}]=-0.57$ electrons. ${ }^{73}$ High-frequency dielectric constants are calculated to be, $\varepsilon_{\|}=15.60$ and $\varepsilon_{\perp}=6.34$ in the intralayer and interlayer directions, respectively. The values calculated from the first principles are in good agreement with those determined by Sun et al. ${ }^{17}$ from experimental data. We note that contour plots of the calculated charge density, $\rho(\mathbf{r})$ indicate that $\mathrm{MoS}_{2}$ layers have directional bonds. These bonds are formed by Mo-d and S-p orbital hybridization and have a significant ionic component. The charge transfer estimated by Mulliken ${ }^{74}$ analysis indicates an excess electronic charge of 0.215 electrons on each $S$ 
(a) 2H-MoS

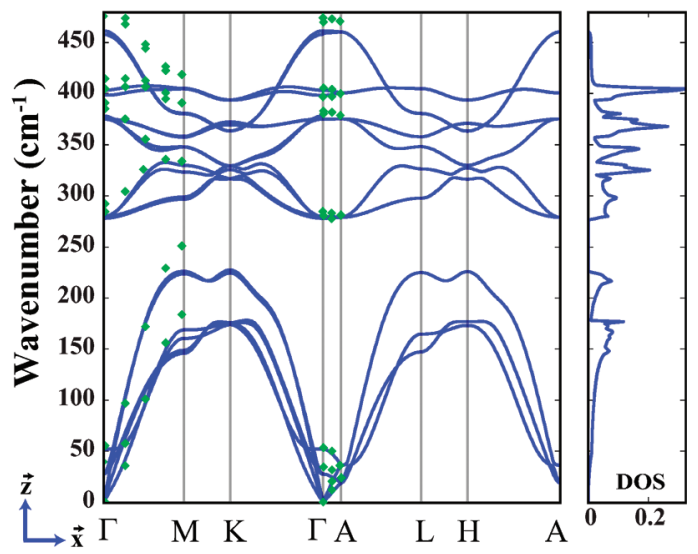

$\begin{array}{llllllll}\mathbf{E}_{2 \mathrm{u}} & \mathbf{E}_{1 \mathrm{~g}} & \mathbf{E}_{2 \mathrm{~g}} & \mathbf{E}_{1 \mathrm{u}} & \mathbf{B}_{1 \mathrm{u}} & \mathbf{A}_{1 \mathrm{~g}} & \mathbf{A}_{2 \mathrm{u}} & \mathbf{B}_{2 \mathrm{~g}}\end{array}$

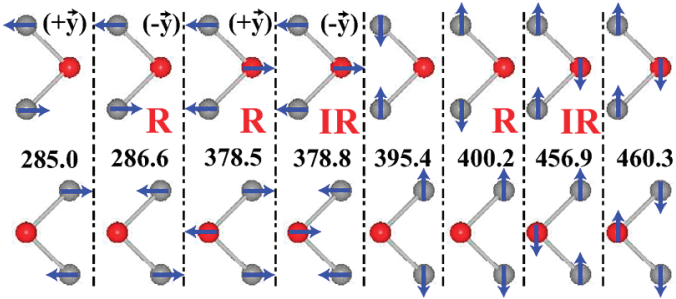

(c)

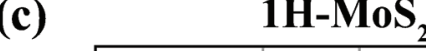

(d)
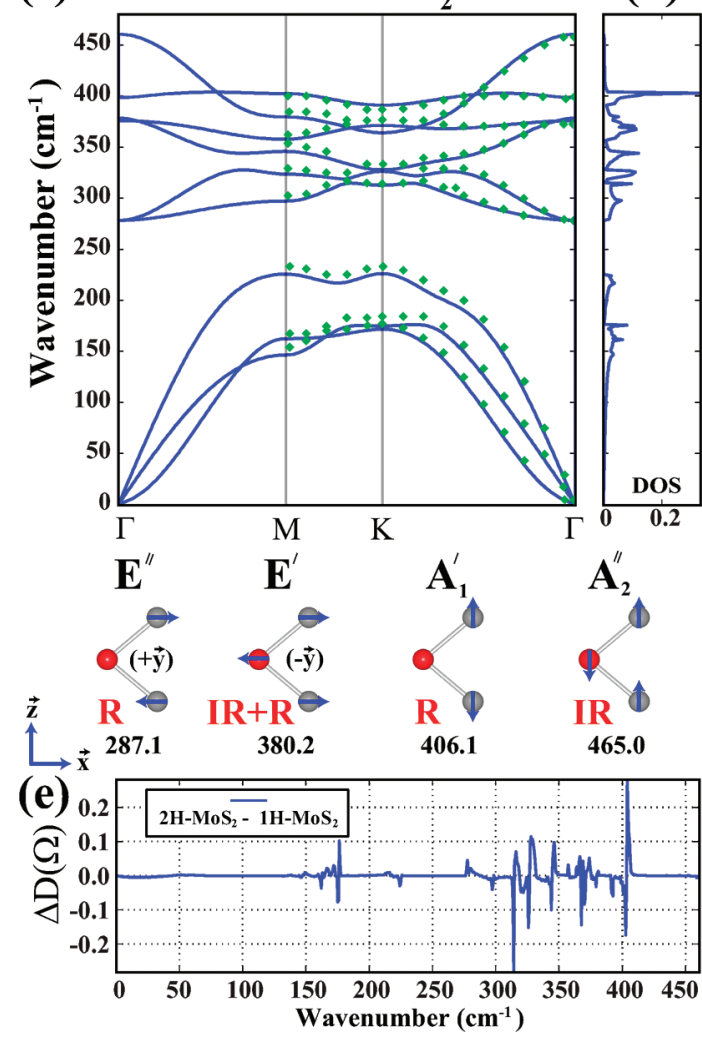

Figure 2. (a) Calculated phonon dispersion curves of $2 \mathrm{H}-\mathrm{MoS}_{2}, \Omega(\mathbf{k})$ versus $k$ along symmetry directions of $\mathrm{BZ}$, and corresponding density of states (b). (c) and (d) are the same as (a) and (b) for $1 \mathrm{H}-\mathrm{MoS}_{2}$. (e) Difference of the densities of states of $2 \mathrm{H}-\mathrm{MoS}_{2}$ and $1 \mathrm{H}-\mathrm{MoS}_{2}$ (see text). Phonon branches derived from neutron scattering data ${ }^{6}$ and branches calculated by using a local basis set ${ }^{46,77}$ are indicated in (a) and (c) by green (light) squares, respectively. Infrared (IR) and Raman (R) active modes with symmetry representations and frequencies $\left(\mathrm{cm}^{-1}\right)$ at the $\Gamma$-point are indicated. atom and depletion of electrons on each Mo atom amounting to 0.430 electrons. Negatively charged $S$ atoms form negative charge layers on both sides of $2 \mathrm{D} \mathrm{MoS}_{2}$. We note the direction of calculated charge transfer is in compliance with the Pauling's electronegativity scale, ${ }^{75}$ as well as with Born effective charges. On the basis of the charge density analysis, $\mathrm{MoS}_{2}$ layers can be viewed as a positively charged Mo atomic plane sandwiched between two negatively charged S-atomic planes. As for $1 \mathrm{H}-\mathrm{MoS}_{2}$, Born effective charges are calculated to be $Z_{\mathrm{B}}{ }^{*}[\mathrm{Mo}]=1.21$ electrons for Mo and $Z_{\mathrm{B}}{ }^{*}[\mathrm{~S}]=-0.57$ electrons for $S$. These values are close to those of $2 \mathrm{H}-\mathrm{MoS}_{2}$. However, high-frequency dielectric constants of $1 \mathrm{H}-\mathrm{MoS}_{2}$, which differ dramatically from $3 \mathrm{D} \mathrm{MoS}$ due to dimensionality effect, are $\varepsilon_{\|}=4.58$ and $\varepsilon_{\perp}=1.26$ in the intralayer and interlayer directions, respectively.

In Table 2 we present structure parameters and related properties calculated for $2 \mathrm{H}-\mathrm{MoS}_{2}$ and $1 \mathrm{H}-\mathrm{MoS}_{2}$ using GGA $+\mathrm{D}$. These are lattice constants $a, c$, internal structural parameters, bulk modulus $B_{0}$ (in-plane stiffness $C$ for $1 \mathrm{H}-\mathrm{MoS}_{2}$ ), cohesive energy $E_{\mathrm{C}}$, Born effective charges, and high-frequency dielectric constants.

\section{LATTICE DYNAMICS}

Previously, the lattice dynamics of $2 \mathrm{H}-\mathrm{MoS}_{2}$ has been investigated both experimentally and theoretically. The phonon dispersion curves and density of states have been obtained by fitting the force constants to experimental data. ${ }^{6,24}$ Meanwhile $2 \mathrm{D}$ sheets of $\mathrm{MoS}_{2}$ including the single layer $1 \mathrm{H}-\mathrm{MoS}_{2}$ were synthesized. ${ }^{18-20,22}$ Our objective is (i) to calculate phonon dispersion curves and total density of states of both $2 \mathrm{H}-\mathrm{MoS}_{2}$ and $1 \mathrm{H}-\mathrm{MoS}_{2}$ from the first principles including vdW correction; (ii) to reveal the dimensionality effects between $3 \mathrm{D}$ and $2 \mathrm{D}$ $\mathrm{MoS}_{2}$; (iii) to provide understanding of phonon anomalies observed in Raman spectra. ${ }^{21,46}$

While Mo atoms in $2 \mathrm{H}-\mathrm{MoS}_{2}$ occupy sites of $D_{3 h}$ symmetry, $\mathrm{S}$ atoms obey $C_{3 v}$ symmetry. The overall symmetry of the crystal is $D_{6 h}$, having 24 symmetry elements and 12 irreducible representations. Four second-order representations involve the lateral (in-plane) displacements of Mo and S atoms, as indicated in Figure 2. First-order representations are coupled with the displacements perpendicular to the layers of atoms or parallel to $z$ axis. Similar observations are also valid for monolayer $1 \mathrm{H}$ $\mathrm{MoS}_{2}$ having $D_{3 h}$ symmetry, 12 symmetry elements and 6 irreducible representations. In order for an irreducible representation to be infrared active mode, it must create a dipole moment in the system. For $2 \mathrm{H}-\mathrm{MoS}_{2}, \mathrm{E}_{1 \mathrm{u}}$ and $\mathrm{A}_{2 \mathrm{u}}$ modes are infrared active. Similarly, $\mathrm{E}^{\prime}$ and $\mathrm{A}_{2}{ }^{\prime \prime}$ modes are infrared active modes of $1 \mathrm{H}-\mathrm{MoS}_{2}$. Raman active modes induce polarization or quadruple moment in the lattice. $A_{1 g}, E_{1 g}$ and $E_{2 g}$ modes are Raman active modes for $2 \mathrm{H}-\mathrm{MoS}_{2}$, so as $\mathrm{A}_{1}^{\prime}, \mathrm{E}^{\prime}$, and $\mathrm{E}^{\prime \prime}$ modes for $1 \mathrm{H}-\mathrm{MoS}_{2}$.

Verble and Wieting ${ }^{2,3}$ related the interlayer interaction in $2 \mathrm{H}$ $\mathrm{MoS}_{2}$ with the splitting of the frequencies of $\mathrm{E}_{2 \mathrm{~g}}$ and $\mathrm{E}_{1 \mathrm{u}}$ modes. In both modes the first layer atoms have similar displacements, but the displacements of the second layer atoms are in opposite direction as indicated in Figure 2. The minute difference between the frequencies of $E_{2 g}$ and $E_{1 u}$ shows that the interlayer vdW interaction in $2 \mathrm{H}-\mathrm{MoS}_{2}$ is small. Wakabayashi et al. ${ }^{6}$ first reported the phonon dispersion of $2 \mathrm{H}-\mathrm{MoS}_{2}$ by neutron scattering. Because of experimental limitations, they only reported 12 phonon branches along $\Gamma-M$ and $\Gamma-A$ directions out of 18 available ones. The error term in their experiments is $\pm \% 5$. Bertrand $^{23}$ reported that surface phonons have frequencies lower 
Table 3. Calculated Frequencies of Raman (R) and Infrared (IR) Active Modes (in $\mathrm{cm}^{-1}$ ) of $2 \mathrm{H}$ - and $1 \mathrm{H}-\mathrm{MoS} \mathrm{S}_{2}$ at the $\Gamma-\mathrm{Point}$ and Their Symmetry Analysis ${ }^{a}$

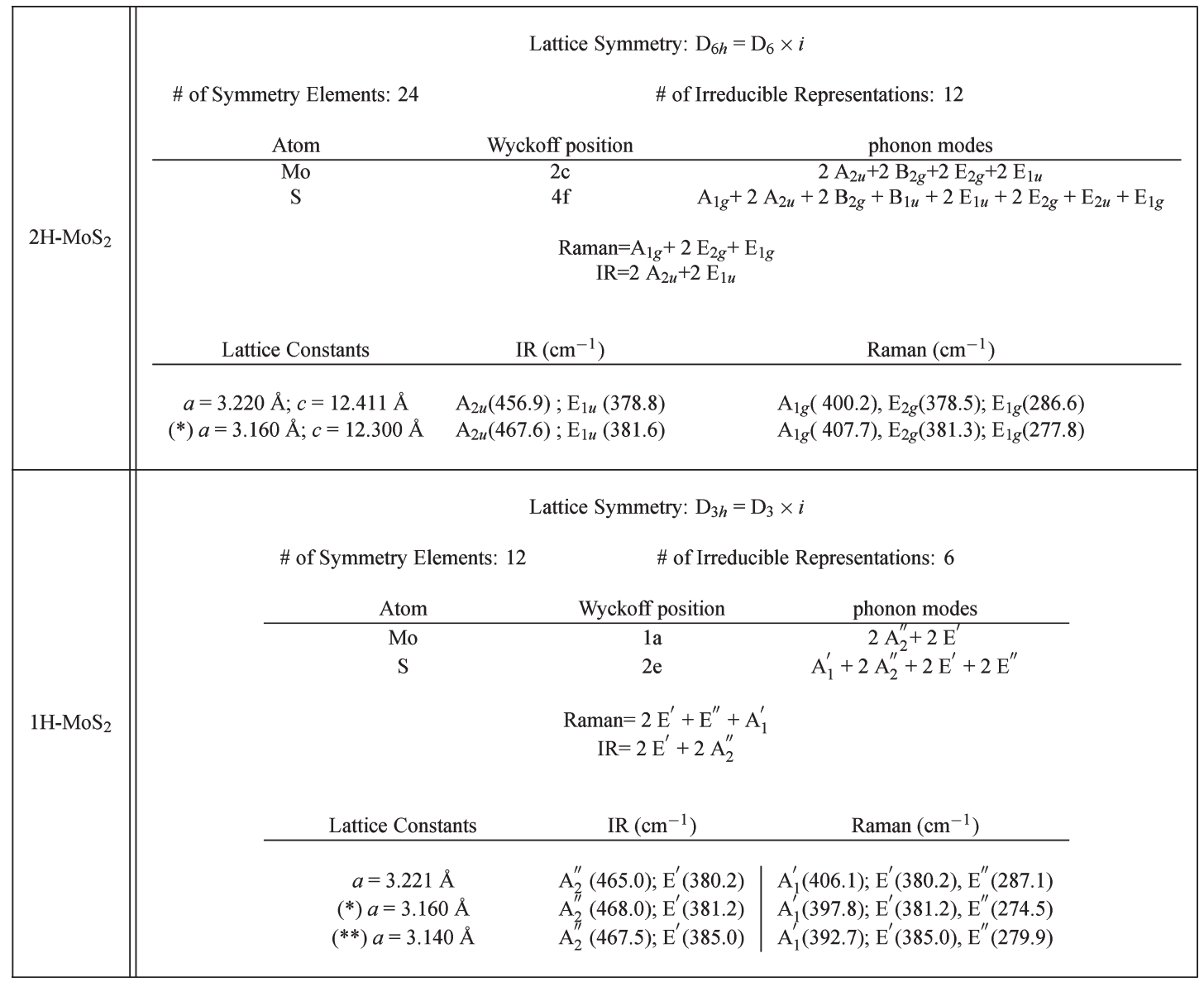

\footnotetext{
${ }^{a}$ The subscripts $\mathrm{u}$ and $\mathrm{g}$ represent antisymmetric and symmetric vibrations, respectively. The other subscript $i(i=1,2,3)$ indicates the stretching modes. IR and $\mathrm{R}$ frequencies of $2 \mathrm{H}$ - and $1 \mathrm{H}-\mathrm{MoS}_{2}$ are calculated for the fully optimized lattice constants and internal structural parameters. Entries of IR and $\mathrm{R}$ frequencies of $2 \mathrm{H}$ - and $1 \mathrm{H}-\mathrm{MoS}_{2}$ indicated by $\left(^{*}\right)$ are calculated using the experimental lattice constants $a$ and $c$ of $2 \mathrm{H}-\mathrm{MoS}_{2}$, but optimizing other internal structural parameters. The entry with $\left({ }^{* *}\right)$ is calculated with $a=3.14 \AA$ and corresponds to $a$, which is smaller than the experimental lattice constant $a$ of $2 \mathrm{H}-\mathrm{MoS}_{2}$.
}

than those of bulk $\mathrm{MoS}_{2}$ phonons. There is a softening of phonon modes upon going to the edges of nanocrystal $2 \mathrm{H}-\mathrm{MoS}_{2}$. Recent experimental study by Livneh and Sterer ${ }^{76}$ revealed the effect of pressure and temperature on the Raman active modes of $2 \mathrm{H}$ $\mathrm{MoS}_{2}$. They reported that upon increase of the temperature of the system, the frequencies of Raman active modes decrease. Whereas the frequencies of Raman active modes increase with increasing pressure.

Phonon dispersion curves of 3D and 2D MoS 2 and their total densities of states calculated within $\mathrm{DFPT}^{55}$ using structure optimized GGA+D are presented in Figure 2. Specific experimental data and earlier calculations are also indicated for the sake of comparison. The phonon branches calculated from the firstprinciples for 3D $\mathrm{MoS}_{2}$ are in overall agreement with experimental data as well as with that calculated using valence force field method. ${ }^{6}$ The calculated acoustical and optical branches of $1 \mathrm{H}-\mathrm{MoS}_{2}, \Omega(\mathbf{k})$ are positive for any $\mathbf{k}$ in BZ. This indicates that the suspended, single layer $1 \mathrm{H}-\mathrm{MoS}_{2}$ structure is stable.

Phonon dispersion curves and corresponding density of states for $2 \mathrm{H}-\mathrm{MoS}_{2}$ and $1 \mathrm{H}-\mathrm{MoS}_{2}$ are similar, except that the number of branches in the former are doubled. Owing to the weak vdW interaction some branches are slightly split. The difference between $2 \mathrm{H}$ - and $1 \mathrm{H}-\mathrm{MoS}_{2}$ is substantiated by the difference of total density of states, i.e., $\Delta D(\Omega)=D_{3 D}(\Omega)-2 D_{2 D}(\Omega)$. The plot of $\Delta D(\Omega)$ in Figure 2e indicates an overall shift of critical point frequencies of $3 \mathrm{D} \mathrm{MoS}$ to slightly higher values, while some modes show a reverse trend. The out of plane acoustical (ZA) mode of $1 \mathrm{H}-\mathrm{MoS}_{2}$ has parabolic dispersion, since the transverse forces decay exponentially. Also the LO-TO splitting is properly predicted. We also determined the infrared (IR) and Raman (R) active modes at the $\Gamma$-point of BZ. Our results presented in Table 3.

Earlier, Raman ${ }^{2,3,9,23,24,78}$ and infrared spectra ${ }^{2,3,17}$ of $2 \mathrm{H}$ $\mathrm{MoS}_{2}$ were studied experimentally. Wieting and Verble ${ }^{2,3}$ reported three Raman active modes at 287,383 , and $409 \mathrm{~cm}^{-1}$. On the other hand, Chen and Wang ${ }^{78}$ have observed four Raman active modes in bulk at $\mathrm{E}_{2 \mathrm{~g}}^{2}=32 \mathrm{~cm}^{-1}, \mathrm{E}_{1 \mathrm{~g}}=286 \mathrm{~cm}^{-1}, \mathrm{E}_{2 \mathrm{~g}}^{1}=$ $383 \mathrm{~cm}^{-1}$, and $A_{1 \mathrm{~g}}=408 \mathrm{~cm}^{-1}$. The small $E_{2 \mathrm{~g}}^{2}$ mode is not observed by Wieting and Verble ${ }^{2,3}$ because of the spectral limit of the Raman measurements between 20 and $1000 \mathrm{~cm}^{-1}$. Also experimentally, at the zone center, IR active modes are observed at $384 \mathrm{~cm}^{-12,3,17}$ and $470 \mathrm{~cm}^{-12,3}\left(468 \mathrm{~cm}^{-117}\right)$. For the case of 
$1 \mathrm{H}-\mathrm{MoS}_{2}$, Lee et al. ${ }^{21}$ investigated the Raman spectra of $2 \mathrm{D}$ $\mathrm{MoS}_{2}$ sheets as a function of thickness down to a single layer $1 \mathrm{H}$ $\mathrm{MoS}_{2}$. They reported that the frequency of the Raman active $\mathrm{A}_{1 \mathrm{~g}}$ mode of $2 \mathrm{H}-\mathrm{MoS}_{2}$ (i.e., thick sheet) decreases gradually from 408 to $\sim 403 \mathrm{~cm}^{-1}$ corresponding to the frequency of $\mathrm{A}_{1}{ }^{\prime}$ of $1 \mathrm{H}$ $\mathrm{MoS}_{2}$ indicating the phonon softening. As for the Raman active mode of $1 \mathrm{H}-\mathrm{MoS}_{2} \mathrm{E}^{\prime}$ displays a reverse behavior and hence its frequency decreases from 383.4 to $382 \mathrm{~cm}^{-1}$ corresponding to the frequency of $\mathrm{E}_{2 \mathrm{~g}}$ mode of $2 \mathrm{H}-\mathrm{MoS}_{2}$. Even if the lateral displacements of atoms in $\mathrm{E}_{2 \mathrm{~g}}$ mode are not affected significantly, one nevertheless expects that all modes of $3 \mathrm{D}$ bulk $\mathrm{MoS}_{2}$ slightly soften in single layer $\mathrm{MoS}_{2}$ due to the absence of interlayer interaction. The observation by Lee et al. ${ }^{21}$ was surprising. In fact, a recent study by Ramakrishna et al. ${ }^{46}$ ended up with a different trend; they observed that both $\mathrm{A}_{1 g}$ and $\mathrm{E}_{2 g}{ }^{\prime}$ modes of $2 \mathrm{H}-\mathrm{MoS}_{2}$ sheets become softer as their thickness decreases.

In this work we attempted to clarify the controversial results reported for the above crucial dimensionality effect. To this end, we calculated the frequencies of Raman active modes of $2 \mathrm{H}-\mathrm{MoS}_{2}$ and $1 \mathrm{H}-\mathrm{MoS}_{2}$ and compared our results with available experimental data. ${ }^{2,3,17,21,46}$ Present GGA+D calculations predict Raman active modes $\mathrm{E}_{1 \mathrm{~g}}=286.6 \mathrm{~cm}^{-1}, \mathrm{E}_{2 \mathrm{~g}}^{1}=378.5 \mathrm{~cm}^{-1}$ and $\mathrm{A}_{1 \mathrm{~g}}=$ $400.2 \mathrm{~cm}^{-1}$ using the optimized lattice constants of $a=3.22 \AA$ and $c=12.41 \AA$. The optimized lattice constant of $1 \mathrm{H}-\mathrm{MoS}_{2}$ did not alter from the optimized value of 3D bulk despite the absence of interlayer interaction. Using the optimized lateral lattice constant $a=3.22 \AA$, we obtained the frequencies of the Raman active modes as $\mathrm{E}^{\prime}=380.2 \mathrm{~cm}^{-1}$ and $\mathrm{A}_{1}^{\prime}=406.1 \mathrm{~cm}^{-1}$. (See Table 3.) According to these results of the ab initio GGA $+\mathrm{D}$ method, the frequencies of both modes should increase as one goes from $3 \mathrm{D}$ to $2 \mathrm{D}$ single layer, which disagree with experimental results, except the behavior of $\mathrm{E}_{2 \mathrm{~g}} \rightarrow \mathrm{E}^{\prime}$ reported by Lee et al. ${ }^{21}$

The source of this disagreement between ab initio calculations and experimental data is sought in the lattice constants, which are overestimated by GGA+D calculations. We repeated the same GGA+D calculations using experimental lattice constants, ${ }^{10,63,64}$ namely, $a=3.16 \AA$ and $c=12.30 \AA$ for $2 \mathrm{H}-\mathrm{MoS}_{2}$ and $a=3.16 \AA$ for $1 \mathrm{H}-\mathrm{MoS}_{2}$ by assuming that the lateral lattice constant, $a$, did not change by going from $3 \mathrm{D}$ to single layer. We find that $\mathrm{A}_{1 \mathrm{~g}}=$ 407.7, $\mathrm{E}_{2 \mathrm{~g}}=381.3 \mathrm{~cm}^{-1}$, and $\mathrm{E}_{1 \mathrm{~g}}=277.8 \mathrm{~cm}^{-1}$ for $2 \mathrm{H}-\mathrm{MoS}_{2}$, while for $1 \mathrm{H}-\mathrm{MoS}_{2} \mathrm{~A}_{1}{ }^{\prime}=397.8 \mathrm{~cm} \mathrm{~cm}^{-1}, \mathrm{E}^{\prime}=381.2 \mathrm{~cm}^{-1}$ and $\mathrm{E}^{\prime \prime}=$ 274.5. Apparently, Raman active modes of $2 \mathrm{H}-\mathrm{MoS}_{2}$ calculated with experimental lattice constants are in good agrement with observed Raman frequencies. ${ }^{2,78}$ Moreover, we are able to reproduce the experimental trend that frequency of the Raman active mode $A_{1 g}$ softens for $A_{1 g} \rightarrow A^{\prime}$, i.e., as the dimensionality is reduced from $3 \mathrm{D}$ to $2 \mathrm{D}$. The change in the frequency is negligibly small for $E_{2 g} \rightarrow E^{\prime}$. Noting the fact that the lattice constant of graphene, $a$, gets slightly smaller than that of $3 \mathrm{D}$ graphite, despite GGA+D optimizes $a$ of $3 \mathrm{D}$ and $2 \mathrm{D}$ almost at the same value. Considering the possibility that the lattice constant of $1 \mathrm{H}-\mathrm{MoS}_{2}$ $a$ can get smaller than the lateral lattice constant of $3 \mathrm{D} \mathrm{MoS}_{2} a=$ $3.16 \AA$, we repeated our calculations for $1 \mathrm{H}-\mathrm{MoS}_{2}$ using $a=3.14 \AA$ and found that the frequency of $E^{\prime}$ increases from 381.2 to $385 \mathrm{~cm}^{-1}$ confirming the anomalous effect reported by Lee et al. (See Table 3.) This is, however, a hypothetical situation and will be clarified when experimental data on the lattice constant $a$ of free-standing $1 \mathrm{H}-\mathrm{MoS}_{2}$ will be available. We also note that Raman active modes of $2 \mathrm{H}-\mathrm{MoS}_{2}$ calculated by LDA, which underestimates the lattice constants was able to reproduce the same dimensionality effect between $3 \mathrm{D}$ and $2 \mathrm{D} \mathrm{MoS}$ as reported by Lee et al., ${ }^{21}$ namely, that while $\mathrm{A}^{\prime}$ softens, $\mathrm{E}^{\prime}$ becomes harder by going from $3 \mathrm{D}$ to $2 \mathrm{D}$. To address the question, whether the Raman active modes of the slabs of $2 \mathrm{D} \mathrm{MoS}$ comply with the above trends, we calculated the frequencies of two-layer and three-layer $\mathrm{MoS}_{2}$ using the experimental value of lattice constant, $a$ of $2 \mathrm{H}-\mathrm{MoS}_{2}$. Since there are no data available for the spacings of layers in slabs, we used again the experimental lattice constant $c$ of $2 \mathrm{H}$ $\mathrm{MoS}_{2}$ and set the spacings equal to $c / 2$. We found that $\mathrm{A}_{1}{ }^{\prime}$ increases with increasing number of layers (namely, $\mathrm{A}_{1}^{\prime} \rightarrow 404.9 \mathrm{~cm}^{-1}$ for bilayer $\mathrm{MoS}_{2}$ and $\mathrm{A}_{1}{ }^{\prime} \rightarrow 405.9 \mathrm{~cm}^{-1}$ for three layer $\mathrm{MoS}_{2}$, and approaches to $\mathrm{A}_{1}{ }^{\prime}$ of $2 \mathrm{H}-\mathrm{MoS}_{2}$. Nevertheless, the absence of experimental data on the structure of bilayer and three layer $\mathrm{MoS}_{2}$ slabs prevents us from drawing more definite conclusions regarding phonon softening or phonon hardening with dimensionality.

Finally, in Table 3 we present the frequencies of the IR active modes calculated using optimized as well as experimental lattice constants. For $2 \mathrm{H}-\mathrm{MoS}_{2}$, the present GGA+D calculations using experimental lattice constants can give values in good agreement with experimental data, namely, $A_{2 u}=467.6 \mathrm{~cm}^{-1}$ (as compared to experimental value of $468 \mathrm{~cm}^{-1}$ ) and $\mathrm{E}_{1 \mathrm{u}}=381.6 \mathrm{~cm}^{-1}$ (as compared to experimental value of $\left.384 \mathrm{~cm}^{-1}\right), 2,17$

\section{DISCUSSION AND CONCLUSIONS}

In this paper we investigated lattice dynamics of 3D layered $\mathrm{MoS}_{2}$ and 2D single layer $\mathrm{MoS}_{2}$. We revealed that weak vdW interaction between layers is critical to hold the layers together and to calculate the interlayer spacing within $0.8 \%$ of the experimental value. Therefore, the inclusion of the vdW interaction between the layers of $\mathrm{MoS}_{2}$ is found to be essential for ab initio calculations of energetics and optimized structures, cohesion, and phonon dispersions. We examined two different approaches to include vdW interaction in DFT calculations within GGA approximation. The spacings between $\mathrm{MoS}_{2}$ layers or perpendicular lattice constant, which are critically overestimated by GGA alone, are improved after the inclusion of vdW interaction. As for the lateral lattice constant $a$, it is almost independent of $\mathrm{vdW}$ interaction. Lattice dynamics and related properties, such as phonon dispersions, effective charges, dielectric constants, etc., calculated within GGA combined with $\mathrm{vdW}$ are found to be in overall agreement with various experimental data and with the empirical values derived therefrom. However, the shifts of the frequencies of Raman active modes by going from $3 \mathrm{D}$ to $2 \mathrm{D}$ single layer are found to be very sensitive to the values of lattice constants used in the calculations. For example, the shifts of Raman active modes predicted by using the lattice constants optimized through GGA with vdW correction disagree with experimental data. We showed that by using experimental lattice constants one can reproduce the experimentally observed shifts. In a similar manner, one can obtain the frequencies of infrared active modes, which agree better with experimental data, if the experimental lattice constants are used in the calculations.

\section{AUTHOR INFORMATION}

\section{Corresponding Author}

*E-mail: ciraci@fen.bilkent.edu.tr.

\section{ACKNOWLEDGMENT}

This work is supported by TUBITAK through Grant No. 104 T537 and Grant No. 108T234. S.C. acknowledges TUBA for 
partial support. Part of the computational resources has been provided by TUBITAK ULAKBIM, High Performance and Grid Computing Center (TR-Grid e-Infrastructure). We thank the DEISA Consortium (www.deisa.eu), funded through the EU FP7 project RI-222919, for support within the DEISA Extreme Computing Initiative.

\section{REFERENCES}

(1) Title, R. S.; Shafer, M. W. Phys. Rev. B 1973, 8, 615-620.

(2) Wieting, T. J.; Verble, J. L. Phys. Rev. B 1971, 3, 4286-4292.

(3) Verble, J. L.; Wieting, T. J. Phys. Rev. Lett. 1970, 25, 362-365.

(4) Kasowski, R. V. Phys. Rev. Lett. 1973, 30, 1175-1178.

(5) Mattheiss, L. F. Phys. Rev. Lett. 1973, 30, 784-787.

(6) Wakabayashi, N.; Smith, H. G.; Nicklow, R. M. Phys. Rev. B 1975, 12, 659-663.

(7) Coehoorn, R.; Haas, C.; Dijkstra, J.; Flipse, C. J. F.; de Groot, R. A.; Wold, A. Phys. Rev. B 1987, 35, 6195-6202.

(8) Kobayashi, K.; Yamauchi, J. Phys. Rev. B 1995, 51, 17085-17095.

(9) Frey, G. L.; Tenne, R.; Matthews, M. J.; Dresselhaus, M. S.; Dresselhaus, G. Phys. Rev. B 1999, 60, 2883-2892.

(10) Böker, T.; Severin, R.; Müller, A.; Janowitz, C.; Manzke, R.; Voss, D.; Krüger, P.; Mazur, A.; Pollmann, J. Phys. Rev. B 2001, 64, 235305.

(11) Rydberg, H.; Dion, M.; Jacobson, N.; Schröder, E.; Hyldgaard, P.; Simak, S. I.; Langreth, D. C.; Lundqvist, B. I. Phys. Rev. Lett. 2003, 91, 126402.

(12) Fuhr, J.; Saul, A.; Sofo, J. Phys. Rev. Lett. 2004, 92, 026802.

(13) Ivanovskaya, V. V.; Zobelli, A.; Gloter, A.; Brun, N.; Serin, V.; Colliex, C. Phys. Rev. B 2008, 78, 134104.

(14) Huang, M.; Cho, K. J. Phys. Chem. C 2009, 113, 5238-5243.

(15) Moses, P. G.; Mortensen, J. J.; Lundqvist, B. I.; Norskov, J. K. J. Chem. Phys. 2009, 130, 104709.

(16) Botello-Mendez, A. R.; Lopez-Urias, F.; Terrones, M.; Terrones, H. Nanotechnology 2009, 20, 325703.

(17) Sun, Q.-C.; Xu, X. S.; Vergara, L. I.; Rosentsveig, R.; Musfeldt, J. L. Phys. Rev. B 2009, 79, 205405.

(18) Joensen, P.; Frindt, R.; Morrison, S. Mater. Res. Bull. 1986, 21, 457-461.

(19) Novoselov, K.; Jiang, D.; Schedin, F.; Booth, T.; Khotkevich, V.; Morozov, S.; Geim, A. Proc. Natl. Acad. Sci. U.S.A. 2005, 102, 10451-10453.

(20) Mak, K. F.; Lee, C.; Hone, J.; Shan, J.; Heinz, T. F. Phys. Rev. Lett. 2010, 105, 136805.

(21) Lee, C.; Yan, H.; Brus, L. E.; Heinz, T. F.; Hone, J.; Ryu, S. ACS Nano 2010, 4, 2695-2700.

(22) Helveg, S.; Lauritsen, J. V.; Lægsgaard, E.; Stensgaard, I.; Nørskov, J. K.; Clausen, B. S.; Topsøe, H.; Besenbacher, F. Phys. Rev. Lett. 2000, 84, 951-954.

(23) Bertrand, P. A. Phys. Rev. B 1991, 44, 5745-5749.

(24) Jiménez Sandoval, S.; Yang, D.; Frindt, R. F.; Irwin, J. C. Phys. Rev. B 1991, 44, 3955-3962.

(25) Seifert, G.; Terrones, H.; Terrones, M.; Jungnickel, G.; Frauenheim, T. Phys. Rev. Lett. 2000, 85, 146-149.

(26) Li, Y.; Zhou, Z.; Zhang, S.; Chen, Z. J. Am. Chem. Soc. 2008, 130, 16739-16744.

(27) Lebegue, S.; Eriksson, O. Phys. Rev. B 2009, 79, 115409.

(28) Bollinger, M.; Lauritsen, J.; Jacobsen, K.; Norskov, J.; Helveg, S.; Besenbacher, F. Phys. Rev. Lett. 2001, 87, 196803.

(29) Ataca, C.; Sahin, H.; Akturk, E.; Ciraci, S. J. Phys. Chem. C 2011, $115,3934-3941$.

(30) Ataca, C.; Ciraci, S. J. Phys. Chem. C 2011, 115, 13303-13311.

(31) Rapoport, L.; Bilik, Y.; Feldman, Y.; Homyonfer, M.; Cohen, S.; Tenne, R. Nature 1997, 387, 791-793.

(32) Hinnemann, B.; Moses, P.; Bonde, J.; Jorgensen, K.; Nielsen, J.; Horch, S.; Chorkendorff, I.; Norskov, J. J. Am. Chem. Soc. 2005, $127,5308-5309$.
(33) Jaramillo, T. F.; Jorgensen, K. P.; Bonde, J.; Nielsen, J. H.; Horch, S.; Chorkendorff, I. Science 2007, 317, 100-102.

(34) Lauritsen, J.; Helveg, S.; Laegsgaard, E.; Stensgaard, I.; Clausen, B.; Topsoe, H.; Besenbacher, E. J. Catal. 2001, 197, 1-5.

(35) Lauritsen, J.; Bollinger, M.; Laegsgaard, E.; Jacobsen, K.; Norskov, J.; Clausen, B.; Topsoe, H.; Besenbacher, F. J. Catal. 2004, 221, 510-522.

(36) Lauritsen, J. V.; Kibsgaard, J.; Olesen, G. H.; Moses, P. G.; Hinnemann, B.; Helveg, S.; Norskov, J. K.; Clausen, B. S.; Topsoe, H.; Laegsgaard, E.; Besenbacher, F. J. Catal. 2007, 249, 220-233.

(37) Moses, P. G.; Hinnemann, B.; Topsoe, H.; Norskov, J. K. J. Catal. 2007, 248, 188-203.

(38) Raybaud, P.; Hafner, J.; Kresse, G.; Kasztelan, S.; Toulhoat, H. J. Catal. 2000, 189, 129-146.

(39) Sun, M.; Nelson, A.; Adjaye, J. J. Catal. 2004, 226, 32-40.

(40) Todorova, T.; Prins, R.; Weber, T. J. Catal. 2007, 246, 109-117.

(41) Kline, G.; Kam, K.; Ziegler, R.; Parkinson, B. Sol. Energy Mater. 1982, 6, 337-350.

(42) Wilcoxon, J.; Thurston, T.; Martin, J. Nanostruct. Mater. 1999, 12, 993-997, 4th International Conference on Nanostructured Materials (NANO 98), Stockholm, Sweden, June 14-19, 1998.

(43) Lauritsen, J. V.; Kibsgaard, J.; Helveg, S.; Topsoe, H.; Clausen, B. S.; Laegsgaard, E.; Besenbacher, F. Nat. Nanotechnol. 2007, 2, 53-58.

(44) Lee, C.; Li, Q.; Kalb, W.; Liu, X.-Z.; Berger, H.; Carpick, R. W.; Hone, J. Science 2010, 328, 76-80.

(45) Radisavljevic, B.; Radenovic, A.; Brivio, J.; Giacometti, V.; Kis, A. Nat. Nanotechnol. 2011, 6, 147-150.

(46) Matte, H. S. S. R.; Gomathi, A.; Manna, A. K.; Late, D. J.; Datta, R.; Pati, S. K.; Rao, C. N. R. Angew. Chem., Int. Ed. 2010, 49, 4059-4062.

(47) Splendiani, A.; Sun, L.; Zhang, Y.; Li, T.; Kim, J.; Chim, C.-Y.; Galli, G.; Wang, F. Nano Lett. 2010, 10, 1271-1275, PMID: 20229981

(48) Vanderbilt, D. Phys. Rev. B 1990, 41, 7892-7895.

(49) Perdew, J.; Chevary, J.; Vosko, S.; Jackson, K.; Pederson, M.; Singh, D.; Fiolhais, C. Phys. Rev. B 1992, 46, 6671-6687.

(50) Grimme, S. J. Comput. Chem. 2006, 27, 1787-1799.

(51) Dion, M.; Rydberg, H.; Schröder, E.; Langreth, D. C.; Lundqvist, B. I. Phys. Rev. Lett. 2004, 92, 246401.

(52) Ceperley, D.; Alder, B. Phys. Rev. Lett. 1980, 45, 566-569.

(53) Kresse, G.; Furthmüller, J. Phys. Rev. B 1996, 54, 11169-11186.

(54) Monkhorst, H. J.; Pack, J. D. Phys. Rev. B 1976, 13, 5188-5192.

(55) Quantum-ESPRESSO is a community project for high-quality quantum-simulation software, based on density-functional theory, and coordinated by Paolo Giannozzi. See http://www.quantum-espresso. org and http://www.pwscf.org.

(56) Arellano, J. S.; Molina, L. M.; Rubio, A.; Alonso, J. A. J. Chem. Phys. 2000, 112, 8114-8119.

(57) Girifalco, L. A.; Hodak, M. Phys. Rev. B 2002, 65, 125404.

(58) Baskin, Y.; Meyer, L. Phys. Rev. 1955, 100, 544.

(59) Trucano, P.; Chen, R. Nature (London) 1975, 258, 136.

(60) Zhao, Y. X.; Spain, I. L. Phys. Rev. B 1989, 40, 993-997.

(61) Bosak, A.; Krisch, M.; Mohr, M.; Maultzsch, J.; Thomsen, C. Phys. Rev. B 2007, 75, 153408.

(62) Hanfland, M.; Beister, H.; Syassen, K. Phys. Rev. B 1989, 39, $12598-12603$.

(63) Kam, K.; Parkinson, B. J. Phys. Chem. 1982, 86, 463-467.

(64) Park, K.; RichardsBabb, M.; Freund, M.; Weiss, J.; Klier, K. J. Phys. Chem. 1996, 100, 10739-10745.

(65) Joensen, P.; Crozier, E.; Alberding, N.; Frindt, R. J. Phys. C: Solid State Phys. 1987, 20, 4043-4053.

(66) Yang, D.; Sandoval, S.; Divigalpitiya, W.; Irwin, J.; Frindt, R. Phys. Rev. B 1991, 43, 12053-12056.

(67) Weiss, K.; Phillips, J. M. Phys. Rev. B 1976, 14, 5392-5395.

(68) Murnaghan, F. D. The Compressibility of Media under Extreme Pressures. Proc. Natl. Acad. Sci. U.S.A. 1944, 30, 244.

(69) Batsanov, S. Effects of explosions on materials: modification and synthesis under high-pressure shock compression. High pressure shock compression of condensed matter; Springer-Verlag: New York, 1994. 
(70) Yakobson, B. I.; Brabec, C. J.; Bernholc, J. Phys. Rev. Lett. 1996, 76, 2511-2514.

(71) Reddy, C.; Rajendran, S.; Liew, K. Nanotechnology 2006, 17, 864-870.

(72) Lee, C.; Wei, X.; Kysar, J. W.; Hone, J. Science 2008, 321, $385-388$.

(73) It is known that Born effective charge is a tensor. The reported experimental as well as calculated values are gvien for each atom in the $x y$ plane. The calculated values for the $z$ direction are $Z_{\mathrm{B}}{ }^{*}[\mathrm{Mo}]=0.58$ and $Z_{\mathrm{B}}{ }^{*}[\mathrm{~S}]=-0.31$ electrons for $2 \mathrm{H}-\mathrm{MoS}_{2}$ and very small values for $1 \mathrm{H}$ $\mathrm{MoS}_{2}$.

(74) Mulliken, R. S. J. Chem. Phys. 1955, 23, 1841-1846.

(75) It should be kept in mind that there are ambiguities in calculating charge transfer. In fact, different methods result in different values of charge transfer.

(76) Livneh, T.; Sterer, E. Phys. Rev. B 2010, 81, 195209.

(77) Soler, J.; Artacho, E.; Gale, J.; Garcia, A.; Junquera, J.; Ordejon, P.; Sanchez-Portal, D. J. Phys.: Condens. Matter 2002, 14, 2745-2779.

(78) Chen, J.; Wang, C. Solid State Commun. 1974, 14, 857-860. 